THU0241

LUPUS PROGRESSION IS PREVENTED BY TREATMENT WITH VERDINEXOR, AN INHIBITOR OF THE NUCLEAR EXPORT PROTEIN EXPORTIN-1, BY LIMITING GERMINAL CENTER FORMATION AND DEVELOPMENT OF AUTOREACTIVE ANTIBODY SECRETING CELLS

Javier Rangel-Moreno ${ }^{1}$, Nida Meednu ${ }^{1}$, Douglas Widman ${ }^{2}$,

Savanna Gornisiewicz' ${ }^{2}$, Sharon Tamir ${ }^{2}$, Jennifer H. Anolik' ${ }^{1}{ }^{1}$ University of Rochester School of Medicine, Division of Allergy, Immunology and Rheumatology, Rochester, United States of America; ${ }^{2}$ Karyopharm Therapeutics, Grants Management, Newton, United States of America

Background: Systemic lupus erythematosus (SLE) is a complex autoimmune disease characterized by simultaneous activation of the innate and adaptive arms of the immune system. Recently the nuclear export protein exportin-1 (XPO1) has surfaced as an attractive target for the treatment of SLE and other autoimmune disorders. Selective Inhibitor of Nuclear Export (SINE) compounds are potent, orally available and well-tolerated XPO1 inhibitors. SINE compounds inhibit the nuclear export of over 220 cargoes, and this pleiotropic effect on multiple signaling pathways exerts apoptotic and anti-inflammatory effects, particularly in dampening the NF-K $B$ response. Based on previous studies with different SINE compounds, we decided to define the minimal amount and frequency of verdinexor treatment necessary to maintain disease inhibition after induction therapy. Objectives: The primary objective of this study was to examine the ability of verdinexor to control the formation of germinal centers and limit the numbers of autoreactive antibody secreting cells (ASC) in a NZB/NZW F1 murine model of SLE.

Methods: To evaluate the minimal efficacious dose of SINE compounds in a preclinical recovery model of SLE, cohorts of lupus-prone mice with established disease (elevated anti-dsDNA antibody titer and proteinuria) were dosed with SINE compound or vehicle for eight weeks, at which time treatment groups were stratified, and escalating administration schedules (both dose and frequency) of verdinexor were examined for their ability to control recurrent disease. We used flow cytometry to enumerate dsDNA antibody-secreting cells (ASC) in the spleen and bone marrow and immunofluorescence to visualize germinal centers (GC) in spleen.

Results: We found that following induction therapy, treatment with verdinexor significantly maintained disease inhibition effectively at $7.5 \mathrm{mg} / \mathrm{kg}$ administered weekly. Concomitantly, we observed significantly decreased levels of GC B cells, plasma cells and plasmablast levels in the bone marrow and the spleen 4 weeks after treatment groups were stratified. The potent effect of SINE compound monotherapy on GC and auto-reactive ASC showed that a pronounced elimination of GC and auto-reactive ASC is achieved after 4 weeks of maintenance therapy administered once weekly. When combined with a proteasome inhibitor (PI), short-term verdinexor plus PI treatment resulted in a synergistic effect, resulting in a significant reduction in the number of auto-reactive antibody secreting cells (ASC).

Conclusion: Verdinexor has demonstrated efficacy in a murine induction/ disease inhibition model of SLE by reducing generation, survival and function of auto-reactive immune cells. It is likely that inhibition of the canonical NF-KB pathway underlies verdinexor's inhibitory effect. The reversible impact of SINE compounds on SLE (symptoms return when therapy is removed) provides a potential window of time for immunization of lupus patients. Together, our findings suggest the potential of SINE compounds to have a significant impact on disease progression in SLE.

Disclosure of Interests: Javier Rangel-Moreno: None declared, Nida Meednu: None declared, Douglas Widman Shareholder of: Karyopharm Therapeutics, Employee of: Karyopharm Therapeutics, Savanna Gornisiewicz Shareholder of: Karyopharm Therapeutics, Employee of: Karyopharm Therapeutics, Sharon Tamir Shareholder of: Karyopharm Therapeutics, Employee of: Karyopharm Therapeutics, Jennifer H. Anolik: None declared DOI: 10.1136/annrheumdis-2019-eular.3092

\section{THU0242 MIR-326 OVEREXPRESSION IN T CELLS BREAKS TH17/ TREG BALANCE IN MRL/LPR MICE}

Yuan Xia, Xuan Fang, Nan Xiang, Xiaojuan Dai, LI Jin, LI Xiaomei, Jinhui Tao, LI Xiangpei. The First Affiliated Hospital of USTC, Division of Life Sciences and Medicine, University of Science and Technology of China, Department of Rheumatology and Immunology, Hefei, China

Background: Systemic lupus erythematosus (SLE) is an autoimmune disease involving multiple organs leading to tissue damage ${ }^{1}$. T lymphocytes have been recognized as key contributors to disease pathogenesis ${ }^{2}$. Inflammatory Th17 subsets promote inflammation, while defects in regulatory $\mathrm{T}$ cells (Tregs) lead to unchecked immune responses ${ }^{3}$. Emerging data suggest that single miR species can profoundly alter the phenotype and outcome of immune responses in $\mathrm{SLE}^{4}$.

Objectives: we describe a role for miR-326 in driving deregulation of Th17/Treg balance in lupus mouse and highlight therapeutic advances of miR-326.

Methods: 3 groups of female MRL/lpr mice were injected with lentivirusmiR-326 (LV-326) or lentivirus-miR-326 specific inhibitor (LV-sponge) to increase or inhibit miR-326 expression, respectively, and lentivirus-noencoding (LV-ctrl) as control, 10 mice per group. The percentage of Th17 cells and Tregs in spleen $\mathrm{CD}^{+}{ }^{+} \mathrm{T}$ cells and kidney were detected by flow cytometry. The levels of serum cytokine (IL-17A, IFN- $\gamma$, TGF- $\beta$ ) were detected by CBA. Urinary collections (24 h) were collected and assessed for albumin using ELISA. Immunohistochemistry labeled kidney IL-17A and TGF- $\beta$ expression. Differences between groups were analyzed by SPSS software.

Results: Day 7 analysis of differentiation of Th17 cells and Tregs in spleen after injection of lentivirus showed an increased percentage of Th17 in LV-326 mice, while reduction of percentage of Tregs in these mice. Testing the serum cytokines revealed that the significantly increased levels of IL-17A and IFN- $\gamma$ in LV-326 mice, and decreased in LV-sponge mice, no different has been found in TGF- $\beta$ between the groups. Moreover, LV-326 mice showed higher levels of $24 \mathrm{~h}$ urinary albumin than LVsponge and LV-ctrl mice in day 7 after injection of lentivirus. The lentivirus-miR-326 injection promoted the infiltration of Th17 cells and simultaneously increased the expression of IL-17A in kidney, no different has been found of TGF- $\beta$.

Conclusion: Our data reveal that miR-326 overexpression increased the differentiation of Th17 cells and decreased the differentiation of Tregs, and accelerated the renal injury in MRL/pr mice. These suggest that miR-326 may play a catalytic role in the development of lupus-like changes in lupus model mouse by breaks Th17/Treg balance.

\section{REFERENCES:}

[1] Tsokos, G.C. Systemic lupus erythematosus. N Engl J Med 365, 2110 2121 (2011).

[2] Moulton, V.R. et al. Pathogenesis of Human Systemic Lupus Erythematosus: $A$

[3] Cellular Perspective. Trends Mol Med 23, 615-635 (2017).

[4] Suarez-Fueyo, A., Bradley, S.J. \& Tsokos, G.C. T cells in Systemic Lupus Erythematosus. Curr Opin Immunol 43, 32-38 (2016).

[5] Lam, I.K.Y., Chow, J.X., Lau, C.S. \& Chan, V.S.F. MicroRNA-mediated immune regulation in rheumatic diseases. Cancer Lett 431, 201-212 (2018).

Disclosure of Interests: None declared

DOI: 10.1136/annrheumdis-2019-eular.6591

\section{THU0243 ROLE OF METHIONINE AND ITS TRANSPORTER CD98 IN HUMAN B CELL DIFFERENTIATION AND THE RELEVANCE TO PATHOLOGICAL PROCESSES OF SLE}

Mingzeng Zhang, Shigeru Iwata, Maiko Hajime, Naoaki Ohkubo,

Yasuyuki Todoroki, Hiroko Miyata, Shingo Nakayamada, Yamagata Kaoru, Yoshiya Tanaka. The First Department of Internal Medicine, University of

Occupational and Environmental Health, Kitakyushu, Japan, Kitakyushu, Japan

Background: B cells play an important role in systemic lupus erythematosus (SLE). Several research protocols have focused in recent years on the topic of immunometabolism. Activation of immunocompetent cells depends on rapid synthesis of cell structure components and biomolecules, which requires enormous amounts of energy, nucleic acids and lipids. Amino acids are important ingredients of many metabolic processes. However, the role of amino acids in plasmablast differentiation and their relevance to the pathogenesis of SLE remain elusive.

Objectives: To determine the role of essential amino acids in human B cell differentiation and relevance to the pathogenesis of SLE.

Methods: In the in vitro arm of the study, purified $\mathrm{CD}_{19^{+}} \mathrm{B}$ cells from healthy donors were cultured with TLR7/9 ligand (LOX or $\mathrm{CpG}$ ), IFN- $\alpha$ and $\mathrm{B}$ cell receptor $(\mathrm{BCR})$ cross-linking, in the presence or absence of amino acids. We determined 1) the types of amino acids that are important for PB differentiation, 2) the amino acid transporters that are important for PB differentiation, 3) the main signaling pathway(s) involved in the presence of amino acids, 4) the transcriptional factors used in the presence of amino acids. In the clinical arm of the study, peripheral blood mononuclear cells (PBMCs) were obtained from 24 patients with RA, 35 patients with SLE, and 28 age-matched healthy controls, and 